\title{
Philosophiques
}

\section{Littéralement dépourvu de sens}

\section{Peter McCormick}

Volume 32, numéro 1, printemps 2005

Questions d'interprétation

URI : https://id.erudit.org/iderudit/011063ar

DOI : https://doi.org/10.7202/011063ar

Aller au sommaire du numéro

\section{Éditeur(s)}

Société de philosophie du Québec

\section{ISSN}

0316-2923 (imprimé)

1492-1391 (numérique)

Découvrir la revue

\section{Citer cet article}

McCormick, P. (2005). Littéralement dépourvu de sens. Philosophiques, 32(1), 55-82. https://doi.org/10.7202/011063ar

\section{Résumé de l'article}

Dire précisément ce que signifient littéralement certaines expressions est souvent important. La compréhension satisfaisante de nombreuses expressions normatives en effet, qu'elles soient juridiques, morales, religieuses, poétiques ou autres, suppose de comprendre ce qu'elles signifient à la fois littéralement et non littéralement. Malgré des recherches pourtant sérieuses et durables sur la nature du " sens littéral ", depuis les anciennes théories religieuses jusqu'aux théories linguistiques et philosophiques contemporaines, une explication généralement satisfaisante des significations supposées littérales des phrases normatives peut s'avérer étonnamment insaisissable. À partir des échanges serrés entre Donald Davidson, Michael Dummett et Ian Hacking, j'aborde un cas de compréhension du littéral dans un discours normatif artistiquement représenté qui est tout aussi difficile à éclaircir. À la différence de Davidson, toutefois, je ne me concentrerai pas sur les aspects qui sont les conditions de vérité des significations littérales supposées des contenus propositionnels de phrases bien assurées et présentées de manière littéraire. J'aimerais plutôt attirer une attention renouvelée sur plusieurs aspects problématiques des significations présumées littérales, spécialement dans des phrases interrogatives présentées sous un jour littéraire lors de conversations normatives lourdes d'un poids éthique. 


\title{
Littéralement dépourvu de sens
}

\author{
PETER MCCORMICK
}

Académie internationale de philosophie du Lichtenstein

pjmccormick@yahoo.com

Nous devons abandonner l'idée d'une structure partagée clairement définie que les utilisateurs du langage acquièrent et appliquent ensuite à des cas particuliers. Et nous devrions essayer encore de dire comment la convention, en quelque sens important que ce soit, est impliquée dans le langage.

Donald Davidson

«Tu ne sais rien? Tu ne vois rien? Tu n’as gardé mémoire De Rien?»

Je me souviens

Those are pearls that were his eyes.

«Es-tu vivant, oui ou non? N'as-tu donc rien dans la tête? » ...

Je ne puis rien

Relier à rien.

T. S. Eliot

RÉSUMÉ. - Dire précisément ce que signifient littéralement certaines expressions est souvent important. La compréhension satisfaisante de nombreuses expressions normatives en effet, qu'elles soient juridiques, morales, religieuses, poétiques ou autres, suppose de comprendre ce qu'elles signifient à la fois littéralement et non littéralement. Malgré des recherches pourtant sérieuses et durables sur la nature du «sens littéral», depuis les anciennes théories religieuses jusqu'aux théories linguistiques et philosophiques contemporaines, une explication généralement satisfaisante des significations supposées littérales des phrases normatives peut s'avérer étonnamment insaisissable. À partir des échanges serrés entre Donald Davidson, Michael Dummett et lan Hacking, j'aborde un cas de compréhension du littéral dans un discours normatif artistiquement représenté qui est tout aussi difficile à éclaircir. À la différence de Davidson, toutefois, je ne me concentrerai pas sur les aspects qui sont les conditions de vérité des significations littérales supposées des contenus propositionnels de phrases bien assurées et présentées de manière littéraire. J'aimerais plutôt attirer une attention renouvelée sur plusieurs aspects problématiques des significations présumées littérales, spécialement dans des phrases interrogatives présentées sous un jour littéraire lors de conversations normatives lourdes d'un poids éthique.

ABSTRACT. - Saying just what some expressions mean literally is often important. For understanding satisfactorily many normative expressions, whether legal moral religious poetic or whatever, requires understanding what these normative expressions mean both literally and non-literally. Despite however serious, protracted investigations of the nature of "the literal sense" from ancient religious 
theories to contemporary linguistic and philosophical theories, a generally satisfactory account of what the supposed literal meanings of normative sentences might come to remains surprisingly elusive. Taking my cue from Donald Davidson's closely argued exchanges with Michael Dummett and lan Hacking, I take up a puzzling instance of understanding the literal in artistically represented normative discourse. Unlike Davidson, however, I will focus not on the truth-conditional aspects of the supposed literal meanings of the propositional contents of literarily represented assertive sentences. Rather, I would like to call renewed attention to several puzzling communicative aspects of supposed literal meanings, especially in literarily represented interrogative sentences in ethically charged normative conversations.

\section{Orientations}

Dire précisément ce que signifient littéralement certaines expressions est souvent important ${ }^{1}$. La compréhension satisfaisante de nombreuses expressions normatives en effet, qu'elles soient juridiques, morales, religieuses, poétiques ou autres, suppose de comprendre ce qu'elles signifient à la fois littéralement et non littéralement.

Malgré des recherches pourtant sérieuses et durables sur la nature du «sens littéral ", depuis les anciennes théories religieuses jusqu'aux théories linguistiques et philosophiques contemporaines, une explication généralement satisfaisante des significations supposées littérales des phrases normatives peut s'avérer étonnamment insaisissable.

L'un des fossés les plus importants aujourd'hui dans cette réflexion dépend précisément de la place où pourrait figurer une explication du littéral dans une théorie philosophique générale de la signification linguistique. De façon très grossière, devrions-nous essayer d'expliquer la nature du littéral à l'intérieur d'une théorie de la signification? Ou faudrait-il plutôt tenter de situer la nature du littéral au sein d'une théorie concurrente du vrai ? Les échanges prolongés et minutieusement argumentés de Donald Davidson, Michael Dummett et Ian Hacking offrent un exemple toujours instructif de ces différences.

J'aimerais dans cet article revenir à ce débat et centrer particulièrement mon attention sur la contribution de Davidson, qui mérite un examen plus approfondi. Mon intérêt est de réexaminer d'un œil critique seulement certaines des réflexions détaillées qu'on y trouve quant à la nature du littéral.

À l'exemple de l'article initial de Davidson qui citait de façon fameuse une magnifique expression non littérale d'une $\mathrm{M}^{\mathrm{me}}$ Malaprop en conversation dans l'une des pièces de Sheridan au dix-huitième siècle ${ }^{2}$, j'aborde un cas de

1. Le Shorter Oxford English Dictionary, $5^{\mathrm{e}}$ édition, Oxford, Oxford University Press, 2002 (cité dans la suite $S O E D$ ) décrit le «littéral " comme «ce qui désigne ou appartient à un sens ou une interprétation d'un texte, spécialement la Bible à l'origine, obtenu en prenant les mots dans leur signification première ou habituelle et en appliquant les règles ordinaires de la grammaire, sans mysticisme, allégorie ni métaphore $[\ldots]$ ».

2. Dans E. LePore, dir., Truth and Interpretation: Perspectives in the Philosophy of Donald Davidson, New York, Blackwell, 1986, p. 433-444. Dans le même ouvrage, voir aussi sur 
compréhension du littéral dans un discours normatif artistiquement représenté qui est tout aussi difficile à éclaircir. À la différence de Davidson, toutefois, je ne me concentrerai pas sur les aspects qui sont les conditions de vérité des significations littérales supposées des contenus propositionnels de phrases bien assurées et présentées de manière littéraire. J'aimerais plutôt attirer une attention renouvelée sur plusieurs aspects problématiques des significations présumées littérales, spécialement dans des phrases interrogatives présentées sous un jour littéraire lors de conversations normatives lourdes d'un poids éthique.

Mon point essentiel ne sera qu'une suggestion, une invitation pressante à une réflexion additionnelle quant à la nature du littéral.

\section{Situations}

J'aimerais proposer ici cette idée que la détermination de la signification littérale de certaines énonciations normatives demeure à la fois plus importante et plus difficile, selon différentes perspectives philosophiques, que ce que nombre d'interprétations actuelles du littéral voudraient toujours nous faire croire. C'est par exemple le cas pour la détermination des significations littérales d'expressions essentielles dans certaines représentations littéraires complexes de situations morales.

Quand nous considérons les rôles variés des énonciations normatives dans nos vies, c'est-à-dire les rôles que certaines des expressions centrales de standards normatifs jouent dans des contextes juridiques, artistiques, moraux, religieux ou autres, l'importance de ces énonciations normatives ressort pleinement. Un examen critique montre en effet assez souvent que certaines de ces expressions en viennent à servir d'indications essentielles pour quelques-uns de nos comportements les plus importants.

Pourtant, même si leur importance semble habituellement évidente dans nos vies, il n'est pas aussi évident de déterminer avec précision ce que signifient ces énonciations normatives. En effet, comme l'illustrent abondamment le sens commun et la sagesse proverbiale, nous exprimons nombre d'entre

cet article les commentaires de Ian Hacking, «The Parody of Conversation », p. 447-458, et de Michael Dummett, "A Nice Derangement of Epitaphs : Some Comments on Davidson and Hacking ", p. 459-476. Cet essai très remarqué doit être de nouveau publié dans le volume 5 à paraître des essais de Davidson, Truth, Language and History. De façon intéressante, Davidson fait suivre dans une section spéciale cet essai par d'autres plus tardifs, dans l'ordre suivant : «The Social Aspect of Language» (1994), «Seeing Through Langage» (1997), «Joyce and Humpty Dumpty» (1989), «The Third Man» (1992), et «Locating Literary Language» (1993).

Le titre original de Davidson fait allusion au personnage du dramaturge et politicien anglais du XVIII ${ }^{\mathrm{e}}$, Richard Sheridan, Mme Malaprop, et à ce que le SOED appelle son " usage impropre et ridicule des mots, spécialement lors de la confusion de deux mots qui se ressemblent » dans la pièce de Sheridan de 1775 , Les Rivaux. Nous pourrions peut-être dire que, dans la pièce, le personnage fictif, $\mathrm{M}^{\mathrm{me}}$ Malaprop, a ridiculement mal usé des mots, confondant « dérangement » et "arrangement ", " épitaphes» et "épithètes ». Elle change ainsi la phrase "un bel arrangement d'épithètes» en «un beau dérangement d'épitaphes». 


\section{$58 \cdot$ Philosophiques / Printemps 2005}

elles de manière non littérale la plupart du temps — que ce soit de manière analogue, métaphorique, symbolique ou autrement. Et, comme nous le savons d'expérience, ces expressions non littérales ne font pas ressortir leur signification aussi clairement que leurs formulations littérales sont supposées le faire. De là nos fréquents désaccords à propos de ces significations.

Quand nous ne parvenons pas à résoudre nos fréquents désaccords relativement à la signification de nombreuses énonciations normatives, nous essayons habituellement de revenir à leur signification littérale à partir de leur signification non littérale. Et, au moins en guise de préambule pour nos discussions à venir, ce que nous supposons être le «sens littéral » sera pris en un sens standard : «la signification stricte, non figurative qu'une expression ou une phrase possède dans le langage en vertu du sens que ses mots ont dans le dictionnaire et de la teneur de ses constructions syntaxiques" par exemple 3 .

Or, quand nous nous détournons de l'analyse grammaticale de la signification complexe non littérale de nombreuses énonciations normatives pour examiner leur signification littérale apparemment plus simple au sens standard de ce qui est littéral, nous nous heurtons à de nouvelles difficultés. La difficulté principale, alors, ne s'avère pas tant être le problème assez sérieux de l'expression du sens littéral de certaines énonciations normatives particulières.

Malgré ce qu'un sens standard du littéral pourrait suggérer, la difficulté principale consiste plutôt à comprendre précisément ce qu'est la signification littérale elle-même. De là les juristes, les linguistes, les philosophes du langage, les critiques littéraires, les exégètes bibliques, les poètes. Et bien d'autres qui en viennent tôt ou tard à s'inquiéter, si ce n'est à se disputer, à propos précisément de ce qu'est la signification littérale. Peut-être serait-il donc justifié d'examiner plus attentivement au moins ce que disent aujourd'hui certains linguistes et philosophes quant à la nature du sens littéral.

\section{Ce qu'est la signification littérale au dire de certains linguistes et philosophes}

Nous pourrions raisonnablement commencer à examiner ce qu'est la signification littérale en considérant ce que certains linguistes et philosophes affirment de son essence dans le cadre d'une expression linguistique. L'œuvre de Donald Davidson constitue un exemple excellent et représentatif d'une telle réflexion ${ }^{4}$.

Dans son article intitulé A Nice Derangement of Epitaphs, Davidson distingue plus spécifiquement, en partant d'une large réflexion linguistique et philosophique sur la nature du littéral, cinq aspects centraux de la signification

3. Voir l'article clair de B. Loar, "Meaning", dans R. Audi, dir., The Cambridge Dictionary of Philosophy, 2e éd., Cambridge, Cambridge University Press, 1999, p. 545.

4. Davidson est actuellement engagé dans le processus d'édition de son œuvre en cinq volumes à Oxford Clarendon Press. Ces volumes incluent les Essays on Actions and Events (1980) originellement publiés et maintenant augmentés ainsi que Inquiries into Truth and Interpretation (1984), Subjective, Intersubjective, Objective (2001) et Problems of Rationality et Truth, Language and History, à paraître. 
littérale auxquels une énonciation doit satisfaire. Bien que Davidson préfère désigner ce qui est traditionnellement dénommé « signification littérale » par ce qu'il appelle ici «sens premier ", je garderai par la suite l'expression plus traditionnelle. Je pense que nous pourrions par anticipation formuler sans injustice ces cinq aspects comme suit.

Nous pourrions tout d'abord bien considérer que le concept de la signification littérale d'une expression linguistique est l'ensemble des usages réels "normaux » ou «standards » d'une énonciation, tels qu'ils sont présentés par un dictionnaire détaillé.

La signification littérale d'une expression linguistique est en second lieu constituée par le sens et la portée d'une énonciation tels qu'ils sont d'abord envisagés par le locuteur. Plus spécifiquement, la signification littérale d'une expression linguistique comprend les propriétés sémantiques relationnelles des éléments et structures d'une énonciation.

Quatrièmement, la signification littérale d'une expression linguistique est simplement ce qui est communiqué "avec succès et régulièrement " par le locuteur à un interprète (un auditeur, un lecteur et ainsi de suite).

Finalement, la signification littérale d'une expression linguistique est un aspect conventionnel d'une énonciation gouvernée par des régularités qu'un locuteur et un interprète ont apprises auparavant de façon suffisante et systématique.

Nous pourrions considérer cette quintuple description de la signification linguistique littérale comme un standard très représentatif de ce qu'est la signification littérale pour les linguistes et les philosophes d'aujourd'hui et donc la qualifier d'« explication standard».

\section{Ce qu'il en coûte de s'occuper d'une difficulté de l'explication standard}

Nous devrions noter que Davidson lui-même a utilisé le titre de son article pour faire ressortir une difficulté plutôt sérieuse à laquelle doit faire face toute explication standard de ce type de signification littérale.

Dans le titre de son article et en général, Davidson soutient en détail qu'une explication standard de la signification littérale ne peut pas expliquer, comme elle le devrait, la nature de la signification littérale de certaines énonciations linguistiques telles que les impropriétés de langage. L'une de ses nombreuses préoccupations essentielles est alors de renforcer cette explication standard de la signification littérale de sorte qu'elle puisse expliquer comment les interprètes parviennent à comprendre la signification littérale d'expressions anormales comme les impropriétés de langage.

Il introduit une distinction afin d'identifier précisément ce qui requiert une explication plus approfondie dans le contexte d'une conversation, par exemple dans le cas où un locuteur use d'impropriétés de langage et où, malgré l'occurrence de cette expression anormale, l'interlocuteur réussit pourtant à comprendre littéralement non pas seulement ce qui a été dit en fait, mais ce qu'on avait essentiellement l'intention de dire. 
La distinction s'établit entre la structure préalable et la structure passagère de la compréhension linguistique. Ce qui est décisif pour l'interprète dans sa compréhension littérale de la signification du locuteur, c'est l'intention communicative principale qui accompagne l'usage d'une expression anormale par le locuteur. Pour continuer à s'adapter à cette intention, l'interprète a besoin de modifier sans cesse sa structure linguistique passagère. Et, au fur et à mesure que la conversation avance, l'interprète doit agir ainsi de façon à continuellement mettre à jour sa propre structure linguistique, susceptible de correspondre à la structure linguistique préalable du locuteur, en constant changement.

Davidson croit que cette distinction pourrait nous permettre d'expliquer la manière dont un locuteur parvient à communiquer la signification littérale de son intention principale, bien que celui-ci use d'expressions anormales dont les signification littérales sont antérieurement inconnues à son interlocuteur.

L'investissement consenti pour cette distinction finit toutefois par être coûteux. Davidson croit aussi en effet que cette distinction sape de manière décisive la compréhension tout à fait fondamentale de la compétence linguistique qui sous-tend les troisième et quatrième aspects de l'explication standard de la signification littérale.

Or, après réflexion critique, la distinction s'avère plus fiable que les aspects en question. Davidson se retrouve donc à la fois avec son problème initial de savoir comment élucider le littéral de façon plus satisfaisante que ce que semblerait permettre l'explication usuelle imparfaite et avec le nouveau problème, plus fondamental, d'une explicitation nouvelle de ce à quoi aboutit précisément de façon standard la compétence linguistique.

Davidson écrit : "Dans la communication linguistique rien ne correspond à la compétence linguistique telle qu'elle est souvent décrite ", c'est-àdire telle qu'elle est envisagée plus haut aux points trois, quatre et cinq de l'explication standard de la signification littérale. Bien que la compréhension de la compétence linguistique qui sous-tend les points trois et quatre puisse rester valable si l'on prend des «voies inhabituelles» pour les modifier de façon radicale, la compréhension de la compétence linguistique qui sous-tend le dernier point, Davidson en est convaincu, «ne peut pas être valable». Et « on ne voit pas bien ce qui pourrait la remplacer».

Sur ces bases, Davidson abandonne la compréhension de la compétence linguistique en jeu. Puisqu'il croit que nous ne pouvons comprendre une langue sans saisir ce à quoi ressemble une explication satisfaisante de la compétence linguistique, et étant donné qu'il rejette l'explication standard sans offrir d'alternative raisonnable, il conclut son article de façon fameuse et dramatique.

«Je conclus ", écrit-il, qu'il n'y a rien de tel qu'une langue, si une langue est quoi que ce soit de ressemblant à ce que de nombreux philosophes et linguistes ont supposé. Il n'y a donc rien de tel qui devrait être appris, maîtrisé ou bien avec quoi il faudrait naître. Nous devons abandonner l'idée d'une structure partagée clairement définie que les utilisateurs du langage acquièrent 
et appliquent ensuite à des cas particuliers. Et nous devrions essayer encore de dire comment la convention, en quelque sens important que ce soit, est impliquée dans le langage; ou bien, comme je le pense, nous devrions abandonner la tentative d'éclairer la façon dont nous communiquons en faisant appel à des conventions (446).»

\section{Une approche moins coûteuse de la signification littérale?}

Je ne suis pas le seul à penser que quelque chose ici semble être allé de travers. Mais à la différence d'autres personnes comme Michael Dummett et Ian Hacking, je ne crois pas que le problème principal réside ici dans les explications de Davidson sur la manière exacte de comprendre ce qu'est une théorie ou une structure linguistique, ou le rôle de la récurrence dans cette théorie ou cette structure, ou même l'articulation proprement dite de ce que sont précisément une théorie "préalable» et une théorie "passagère ».

Je pense plutôt qu'une difficulté essentielle dépend simplement de ce à quoi aboutit la compréhension de la signification littérale d'expressions linguistiques dans certains échanges conversationnels.

Nous pouvons peut-être éviter la conclusion radicale de Davidson citée plus haut si l'on considère certains cas raisonnablement contrôlables de communication linguistique avortée, un cas par exemple où une conversation semble échouer parce qu'un interlocuteur ne parvient pas à comprendre la signification littérale d'une expression essentielle dans une des énonciations particulièrement importantes du locuteur. Peut-être pourrions-nous même entrevoir une compréhension moins problématique de la signification littérale qui a au premier chef partiellement conduit à cette conclusion radicale.

\section{Le vent sous la porte}

Voici un passage tiré d'un chef-d'œuvre du modernisme littéraire, un poème qui partage avec une grande part de l'œuvre moderniste une représentation verbale tragique des nombreuses relations toujours difficiles à éclaircir entre l'art et la vie ${ }^{5}$. En confrontant aujourd'hui encore des lecteurs sensibles à un

5. T. S. Eliot, "'The Waste Land' : Authoritative Text, Contexts, Criticism », M. North, dir., New York, Norton, 2001, p. 9. Voir J. C. C. Mays, «Early Poems from 'Prufrock' to 'Gerontion' ", dans A.D. Moody, dir., The Cambridge Companion to T. S. Eliot, Cambridge, Cambridge University Press, 1994, p. 109-110.

Relativement à certains détails historiques et biographiques pertinents qui concernent les relations d'Eliot avec sa première femme, Vivienne Haigh-Wood (dont le frère, non pas Eliot lui-même — n'en déplaise à certains critiques littéraires, à la pièce de Michael Hasting en 1984, «Tom et Viv », et au film de 1994 - la plaça dans un asile en 1938), voir, parmi d'autres écrits plus approfondis, l'article récent de L. Menand «The Women Come and Go : The Love Song of T. S. Eliot» dans le New Yorker du 30 septembre 2002. Quant à savoir si la conversation fictive confuse de «La Terre vaine » que je cite est en quelque façon une représentation littérairement transposée d'un fragment de la vie personnelle d'Eliot et Vivienne, cela ne peut être que spéculation. J'indique donc ce point en utilisant parfois l'expression "le poète » au lieu de toujours dire « Eliot». 


\section{2 - Philosophiques / Printemps 2005}

sentiment de souffrance profondément personnelle qui transcende pourtant la sphère purement individuelle, cette œuvre poétique d'art verbal continue à soulever des questions quant à la manière dont nous devons proprement comprendre les significations littérales de questions aussi curieuses sur "rien », dans un contexte qui n'est pas seulement poétique mais aussi moral.

'My nerves are bad to-night. Yes, bad. Stay with me.

'Speak to me. Why do you never speak. ${ }^{6}$ Speak.

'What are you thinking of? What thinking? What?

'I never know what your are thinking. Think.'

I think we are in rats' alley

Where the dead men lost their bones.

'What is that noise?'

The wind under the door.

'What is that noise now? What is the wind doing?'

Nothing again nothing.

'Do

'You know nothing? Do you see nothing? Do you remember

'Nothing?'

I remember

Those are pearls that were his eyes.

'Are you alive, or not? Is there nothing in your head?'

"J'ai les nerfs à vif ce soir. À vif, te dis-je. Reste avec moi.

«Mais parle-moi! Jamais tu ne me parles. Parle.

"A quoi peux-tu penser? À quoi ? Que penses-tu?

«Ah! je ne sais jamais ce que tu penses. Pense.»

Je pense que nous sommes dans l'impasse aux rats

Où les morts ont perdu leurs os.

"Quel est ce bruit?»

C'est le vent sous la porte.

"Qu'est-ce encore que ce bruit? Que peut bien faire le vent?»

Rien. Toujours rien.

"Comment!

«Tu ne sais rien? Tu ne vois rien? Tu n'as gardé mémoire

De Rien ?»

Je me souviens

Those are pearls that were his eyes.

«Es-tu vivant, oui ou non? N'as-tu donc rien dans la tête?»

6. Curieusement, la meilleure des quatre premières éditions de son poème, celle de décembre 1922 publiée à Londres par Boni et Livewright après les éditions d'octobre et novembre, omet le point d'interrogation que l'on peut présumer requis, point d'interrogation qu'incluent les trois autres premières éditions. Voir North, p. xiii. 
Dans ce fragment fictif et poétique représentatif, que devons-nous maintenant comprendre et interpréter comme étant la signification littérale des usages littérairement dépeints du mot « rien » (nothing) ? Cette question soulève plusieurs problèmes différents. En voici quelques-uns seulement.

L'un des emplois de "rien » serait-il ici littéral tandis que les autres seraient non littéraux ${ }^{7}$ ? Quelle est la pertinence philosophique de ces emplois variés de la même expression survenant dans une conversation exposée sous des dehors littéraires ${ }^{8}$ ? Y a-t-il vraiment une pertinence proprement philosophique pour l'usage d'expressions telles que "rien ", que ce soit ici ou ailleurs ${ }^{9}$ ? Chacun des deux interlocuteurs représentés emploie-t-il la même expression dans une phrase pour poser un acte linguistique d'un certain type avec un certain contenu ${ }^{10}$ ?

Chacun des interlocuteurs utilise-t-il la même expression de sorte à présenter la même gamme de propriétés significatives ${ }^{11}$ ? Les deux interlocuteurs utilisent-ils cette expression de manière identique ${ }^{12}$ ? Quelle est exactement la pertinence, à supposer qu'il y en ait une, de ces considérations linguistiques et philosophiques pour l'interprétation raisonnable de cette situation de

7. Pour un panorama linguistique général de différentes questions sémantiques sur la signification, voir, par exemple, "Semantics : The Study of Linguistic Meaning ", dans A. Akmajian et al., dir., Linguistics, $5^{\mathrm{e}}$ éd., Cambridge (Mass.), MIT Press, 2001, p. 227-264. Sur les significations littérales en particulier, voir, par exemple, la discussion de l'hypothèse de la force littérale par opposition à la signification figurative dans S. C. Levinson, Pragmatics, Cambridge, Cambridge University Press, 1983, p. 165-166, 263-265 et 274-276.

8. Que l'on compare, par exemple, les vues de H. P. Grice et J. Searle en 1975 dans, respectivement, "Logic and Conversation " et "Indirect Speech Acts ", réimprimés dans A. P. Martinich, dir., The Philosophy of Language, 4 éd., Oxford, Oxford University Press, 2001, p. 165-175 et p. 176-189. Deux ans avant sa mort, en 1989, Grice examinait encore les problèmes-clés entre lui et ses critiques tels que Searle dans "Retrospective Epilogue ", dans Studies in the Way of Words, Cambridge (Mass.), Harvard University Press, 1989, p. 339-385, spécialement p. 349-359 sur la signification du mot à l'encontre de la notion, cruciale pour Grice, d'une signification du locuteur.

9. Que l'on se rappelle la controverse toujours célèbre entre Carnap et Heidegger à partir de 1929 quant à savoir quel sens, le cas échéant, on pourrait attribuer à certaines affirmations heideggeriennes telles que : la science ne connaît "rien à rien" (comparer le vers du poète : "N'as tu donc rien dans la tête?»). Bien que sujette à controverse, une très bonne discussion récente, avec toutes les références, peut être trouvée in S. Critchley, Continental Philosophy, Oxford, Oxford University Press, 2001, p. 90-110.

10. Voir, par exemple, W. P. Alston, Illocutionary Acts and Sentence Meaning, Ithaca, Cornell University Press, 2000, p. 275-309.

11. Que l'on considère, par exemple, les sept «contraintes» que P. Horwich explique sous les rubriques de la compréhension, de l'expression, de la représentation, de l'aprioricité, de la "compositionnalité", de la normativité et de l'usage dans son ouvrage Meaning, Oxford, Clarendon Press, 1998, p. 12-42. Voir aussi la discussion de A. Berger dans son ouvrage Terms and Truth, Cambridge (Mass.), MIT Press, 2002, p. 37-40, quant à la question de savoir si l'expression "masse» en physique newtonienne réfère à la même entité que la "masse au repos » (rest mass) dans la théorie de la relativité.

12. Voir, par exemple, A. Cruse, «Paradigmatic Sense Relations of Inclusion and Identity» dans Meaning in Language, Oxford, Oxford University Press, 2000, p. 146-160. 
communication avortée curieuse, quoique bien familière après tout, poétiquement représentée dans ce dialogue incomplet ${ }^{13}$ ? Et ne commettons-nous pas une erreur fondamentale en essayant de comprendre les interfaces entre langage et signification, en mettant trop en relief les rôles de communication du langage ${ }^{14}$ ?

Des questions comme celles-ci invitent dans leur diversité à une réflexion renouvelée eu égard à la viabilité des explications standards des significations littérales pour traiter des expressions non métaphoriques. Si ce n'est pas le cas dans des contextes aussi compliqués que ceux de la vie quotidienne, alors peut-être le sera-ce dans celui de la poésie moderniste, à l'image de la poésie de la souffrance d'Eliot qui transcende le plan simplement philosophique. Comme on aura pu l'avoir déjà pressenti, ces sortes de questions soulèvent encore en effet des problèmes périphériques toujours irrésolus quant à la question suivante : savoir si une explication satisfaisante de ce qui rend à la fois rationnelle et raisonnable une interprétation des sens littéraux d'expressions (si ce n'est de phrases) cruciales devrait accorder la priorité à une théorie de la signification ou à une théorie de la vérité.

Nous ferons bien de reprendre un certain nombre de réflexions philosophiques contemporaines sur la signification si nous devons mieux saisir certains problèmes cruciaux à propos du sens et de la signification dans les conceptions de la raison et de la rationalité qui guident souvent l'interprétation de ces exemples centraux de la poésie moderniste comme The Waste Land ( «La Terre vaine»).

Que l'on se rappelle toutefois que cette citation n'est qu'une représentation poétique et littéraire d'une conversation incomplète et avortée. Considérons brièvement plusieurs usages de l'expression «rien ", qui diffèrent de manière importante. Nous essayerons ensuite de décrire ce qui semble ici en cause en nous penchant attentivement sur certaines observations de Davidson, avant de rassembler quelques éléments du contexte et de l'information d'arrière-plan.

\section{Quelques usages de «rien »}

Un homme est tout d'abord présenté, qui emploie le mot «rien » sans guillemets. Le poète, toutefois, ne présente pas cet homme comme essayant de dénoter le rien par opposition à la dénotation du mot «rien". Lorsque le poète représente l'homme comme usant du mot «rien » sans guillemets, il le représente plutôt ne répondant que mentalement et non à voix haute aux questions répétées d'une femme.

13. Voir, par exemple K. Bach et R. M. Harnish, «Linguistic Communication : A Schema for Speech Acts », dans S. Davis, dir., Pragmatics : A Reader, New York, Oxford University Press, 1991, p. 231-241.

14. Voir, par exemple, N. Chomsky, On Nature and Language, Cambridge, Cambridge University Press, 2002, p. 121-122. 
Ensuite, comme si elle avait d'une certaine façon entendu tout haut ce que l'homme n'avait prononcé que de façon silencieuse dans sa tête, le poète présente cette femme en train de souligner le mot «rien » en le répétant trois fois à haute voix. Qui plus est, le poète représente cette femme en train de répéter le mot comme si ces répétitions devaient être prises comme constitutives d'une sorte de reproche à l'homme pour ses nombreuses incapacités supposées — «Tu ne sais rien? Tu ne vois rien? Tu n'as gardé mémoire/De Rien?».

Le poète sélectionne aussi attentivement la longueur des lignes, l'espacement, la ponctuation et l'impression pour mettre le mot «rien » en relief. Il le place seul sur une ligne bien à lui. Il enferme maintenant le mot dans des guillemets (à la différence des mots silencieux de l'homme). Et il utilise une majuscule pour la première lettre du mot (de même que le poète a aussi utilisé plus haut une majuscule pour la même première lettre du premier mot dans l'énonciations mentale de l'homme en trois mots).

Le poète continue et représente l'homme qui ne répond que mentalement une fois encore aux questions de la femme en citant, sans guillemets : Those are pearls that were his eyes, une réplique tirée de "La Tempête". Cette réplique est empruntée à un discours de l'Ariel de Shakespeare à propos du père de Ferdinand qui s'est apparemment noyé, à propos de quelqu'un dont le corps est censément décomposé dans la mer, comme celui d'un des personnages fictifs d'Eliot dans le poème, ou peut-être même dans la vie, comme celui de son ami, Jean Verdenal, «mort aux Dardanelles».

Le poète représente finalement la femme qui questionne une fois encore l'homme, d'un air réprobateur, comme si elle avait entendu une fois de plus tout haut ce qu'il n'avait exprimé que silencieusement dans sa tête. "Es-tu vivant, oui ou non?", demande-t-elle (c'est moi qui souligne). Et le poète continue en représentant la femme qui rompt alors complètement et amèrement ses échanges conversationnels déjà fortement fragmenté avec cet homme dont la tête a été pleine de songeries silencieuses, de réponses subtiles, et même de citations littéraires. Bien que cela fût demeuré inexprimé, elle semble en avoir deviné la majeure partie. Elle rompt l'échange avec l'insulte mordante, presque métaphysique "N'as-tu donc rien dans la tête?».

Certains lecteurs pourraient demander ce que nous devons précisément comprendre par la signification littérale de «rien » dans la question "N'as-tu donc rien dans la tête?", question représentée sous un jour poétique et dramatique.

\section{Décrire la situation représentée}

Si nous sommes bien là face à certains emplois essentiels de l'expression "rien ", quelle est la situation représentée ici par le poète ? Elle s'apparente à une situation familière à nombre de gens, dans la vie comme dans l'art.

Ainsi, la situation ressemble ici à une représentation littéraire véritablement poétique d'une scène chargée sur le plan éthique d'un cas de souffrance personnelle intense. Cette souffrance n'est pas nécessaire, et c'est 
pourquoi elle est moralement répréhensible. Or elle se situe des deux côtés d'un échange conversationnel avorté entre un homme et une femme représentés sur un mode littéraire dans un dialogue poétique et dramatique.

D'un côté, une femme semble harceler sans cesse un homme dans la vaine tentative de l'aiguillonner au moins à dire quelque chose. Dirait-il seulement quelque chose qu'il répondrait par là même aux peurs explicites de cette femme et aux anxiétés bien plus substantielles, quoique implicites, qu'elles représentent.

Et, de l'autre, un homme semble bien trop résolument demeurer silencieux. Il refuse de répondre et de dire un seul mot à voix haute. Il semble même essayer de faire taire ses monologues intérieurs fragmentés avec d'obscures répétitions mentales du mot "rien » et ses associations. Toujours est-il que l'homme est représenté ici comme apportant des réponses inexprimées aux questions impératives répétées de la femme — «Mais parle-moi! Jamais tu ne me parles. Parle.»

Le poète présente sans guillemets les réponses inexprimées de l'homme aux questions insistantes de la femme de manière à manifester graphiquement que les réponses de l'homme sont purement mentales. De plus, ces réponses muettes sont pleines d'un désespoir allusif tant historique que littéraire, et peut-être même plus large, à la suite de la Première Guerre mondiale où l'ami parisien d'Eliot, Jean Verdenal, fut tué en 1915, et après ses propres études de la tragédie vengeresse de Shakespeare et Jacob — «Those are pearls that were his eyes".

Le poème transpose lentement ce désespoir qui dépasse le domaine simplement littéraire à l'intérieur d'une sphère publique et extérieure. Une appréhension limitée d'une souffrance personnelle très profonde, privée et finalement incommunicable, quoique partagée, y devient en partie disponible pour que d'autres en soient les témoins et y réfléchissent. La femme souffre de ne recevoir aucune réponse verbale à ses appels répétés à l'homme. Et l'homme souffre de ne pas parvenir à trouver un stratagème mental efficace pour se disculper pleinement sur le plan moral de son refus persistant et résolu de répondre à des appels à l'aide répétés et toujours plus urgents.

Curieusement, le poète parvient néanmoins à représenter les réponses silencieuses de l'homme au harcèlement verbal de la femme comme se communiquant de façon si vive qu'elles donnent naissance à des questionnements toujours nouveaux et obstinés de la part de cette femme. Puis, soudainement, c'est elle qui rompt entièrement la conversation déjà incomplète par une série saccadée de questions toujours plus accusatrices, presque épistémologiques et métaphysiques cette fois — « Tu ne sais rien? Tu ne vois rien? Tu n'as gardé mémoire / De Rien ? "... N'as-tu donc rien dans la tête ${ }^{15}$ ?».

15. D'une manière propre à soulever des controverses, le poète anglais Stephen Spender a écrit que ce complexe échange interpersonnel est «exprimé de manière hystérique par la femme, que l'homme n'y répond pas si ce n'est par des pensées incommunicables et terribles, [et] 
Qu'elle soit ou non une question rhétorique, la puissante dernière phrase — «N'as-tu donc rien dans la tête?» — une phrase interrogative dont les significations littérales et non littérales dépendent du sens et de la signification d'un mot difficile, suffit apparemment à apaiser et à faire taire complètement les songeries intérieures de l'homme jusqu'alors incessantes.

Parmi les nombreux éléments bien délicats de ces représentations poétiques de la souffrance, on trouve les significations "littérales ", les usages très délicats de certains types de phrases et d'expressions tout autant ici que dans d'autres poésies modernistes de la souffrance. Les usages variés, peut-être parfois anormaux, de l'expression clé « rien $^{16}$ » que propose ici Eliot constituent en particulier une partie centrale de ce qui demeure profondément difficile à éclaircir. Un moyen traditionnel de traiter de ces questions difficiles concernant les significations supposément littérales d'expressions dans les œuvres d'art littéraires est de jeter un œil rapide à certains éléments du contexte historique et personnel entourant la création de l'œuvre en question.

\section{Le contexte}

À la fin de l'automne 1921, plusieurs années après la Première Guerre mondiale, T. S. Eliot souffre en Angleterre d'une détérioration à la fois physique et mentale de son état.

T. S. Eliot s'effondra après s'être marié précipitamment en 1915 et avoir ensuite été le témoin de la liaison de sa femme avec Bertrand Russell, son ancien tuteur de philosophie à Harvard, après que sa thèse en philosophie sur F. H. Bradley eut été acceptée à Harvard en 1916, et après avoir raté le diplôme pour ne s'être pas présenté à la soutenance ${ }^{17}$. C'était aussi après la perte cruelle, à Gallipoli, de son ami, le jeune médecin français Jean Verdenal,

que c'est là où le poème touche le poète le plus intimement. C'est le saint des saints du poème. C'est le cœur blessé de la souffrance. Le poète est ici cité contre l'étalage du comportement conditionné de la femme qui affirme tout haut : "Que ferons-nous demain? / Que ferons-nous jamais?" Ses commentaires inexprimés possèdent cette immédiateté de la parole pour laquelle le seul langage possible est celui de la poésie» (Eliot, London, Fontana, 1975, p. 108).

16. Dans le passage que j'ai cité, Eliot utilise le mot nothing ( «rien») de façon variée, mais surtout comme nom. Le SOED rapporte que, lorsqu'il est utilisé comme nom, nothing peut être généralement paraphrasé comme : not any (material or immaterial) thing, "aucune chose (matérielle ou immatérielle) ». Et, faisant appel à l'ancien usage anglais en deux mots, no et thing, les éditeurs du SOED continuent en affirmant que nothing peut être paraphrasé positivement comme : not one thing ( "pas une chose»), quand il est «suivi d'un adjectif ou d'une phrase ou proposition limitatives». Techniquement, sur des problèmes spécifiques à propos de ce que les linguistes appellent «le potentiel de négation du changement de contexte », voir G. Chierchia, et S. Connell-Ginet, Meaning and Grammar, $2^{\mathrm{e}}$ éd., Cambridge (Mass.), MIT Press, 2000, p. 374-376. Et à propos de problèmes concernant les liens anaphoriques d'un terme au sein d'une communauté linguistique, voir A. Berger sur la sémantique des formulations existentielles négatives vraies dans son Terms and Truth, Cambridge (Mass.), MIT Press, 2002, p. 150-156.

17. Bien qu'il n'ait jamais été défendu, le doctorat d'Eliot a été publié plus tard sous le titre Knowledge and Experience in the Philosophy of F. H. Bradley, London, Faber and Faber, 1964. 
"mort aux Dardanelles », à qui il avait dédié un premier recueil de poèmes, et après avoir écrit certaines de ses meilleures critiques malgré les affectations antisémites de ses infatuations passagères à l'Action française. Ce fut enfin à cause de la fatigue croissante due aux maladies de sa femme, qui empiraient, et auxquelles les amis continuaient à faire référence de façon trop condescendante en parlant des "nerfs ", à cause des problèmes financiers persistants et de la mort de son père, toujours désapprobateur, loin là-bas à Saint Louis, dans le Missouri.

Il accepta alors à contrecœur le conseil de son médecin et prit du repos. Il se rendit à Margate, la station balnéaire sur l'estuaire de la Tamise. Et il continua à écrire de la poésie.

Mais lorsque ce changement se révéla par trop inadéquat — "On Margate Sands, écrivit-il plus tard curieusement dans "Le sermon du feu" de "La Terre vaine", I can connect/Nothing with nothing" (Sur la plage, à Margate. / Je ne puis rien / Relier à rien), - il quitta l'Angleterre pour la Suisse afin de consulter un spécialiste. Et, alors qu'il était à Lausanne durant le mois de novembre et une partie du mois de décembre 1921, adaptant les mots du psalmiste ( Auprès des eaux du Léman [lac de Genève], je me suis assis et j'ai pleuré »), Eliot compléta les manuscrits de ce qui devait devenir un de ses nombreux chefs-d'œuvre poétiques ${ }^{18}$.

Toutefois, certaines parties de ces manuscrits comprenaient toujours des matériaux abstrus quasi-philosophiques venant des années passées à Harvard où Eliot ne s'était pas contenté d'étudier en profondeur la philosophie, mais aussi la grammaire du Sanscrit et également des textes tirés des Upanishad. Et l'intégration correcte de ces obscurs fragments (le poème se termine par une répétition de mots sanscrits auparavant utilisés dans le poème : Datta. Dayadhwam. Damyata./Shantih shantih shantih) s'avéra une part essentielle de la difficulté que rencontra Eliot en amenant le poème à un état d'achèvement satisfaisant ${ }^{19}$.

18. Le psaume 137, une lamentation sur la destruction de Jérusalem, commence ainsi : By the waters of Babylon / there we sat down and there we wept / when we remembered Zion (version standard révisée de la traduction anglaise, 1989). "Au bord des fleuves de Babylone / nous étions assis et nous pleurions, / nous souvenant de Sion " (version française de la Bible de Jérusalem, Paris, Éd. du Cerf, 1973).

19. "Shantih, nous dit Eliot dans la dernière de ses notes célèbres, est la fin solennelle d'une Upanishad. Le mot signifie, continue-t-il, quelque chose comme ce qui est signifié par la parole de la version anglaise traditionnelle de la Bible, dite version du roi Jacques, à propos de "la Paix qui dépassait l'entendement" ". En ce qui concerne les trois mots précédents, la note d'Eliot fait référence à l'Upanishad en question mais ne nous dit malheureusement pas ce que les mots signifient. North commente utilement ces mots en résumant l'histoire de la section intitulée «Les trois grandes disciplines» de l'Upanishad Brihadaranyaka 5.1 comme suit. «[...] Dieu présente la syllabe énigmatique DA à trois groupes de disciples, mettant chaque groupe au défi de la comprendre. Chaque groupe est censé comprendre la syllabe comme la racine d'un impératif différent : damyata ("dirige") pour les dieux qui ne sont naturellement pas soumis à des règles, datta (“donne”) pour les hommes qui sont avares; dayadhvam (“compassion”, c.-à-d., soit compatissant) pour les démons, qui sont cruels» (North, 2001, p. 18, note 3). 
Ensuite, à la fin décembre 1921, Eliot emmena ses manuscrits à Paris pour les montrer à Ezra Pound. Il célébra plus tard l'aide extraordinaire de Pound qui réduisit considérablement le manuscrit, ce qui lui permit ainsi de terminer l'œuvre en dédicaçant le poème achevé à Pound comme il miglio fabbro. Eliot publia finalement le poème en octobre 1922.

Malheureusement, en trois versions qui diffèrent légèrement mais de manière significative, il publia aussi le même poème en trois autres endroits, d'abord en novembre, puis en décembre, et ensuite plus tard en 1923. Et pour aggraver la difficulté, Eliot continua de bricoler ces quatre "premières éditions ", en introduisant d'autres variantes légères, même après la parution des Collected Poems 1909-1962 en 1963 et des Collected Poems and Plays en 1969. Le résultat en fut qu'aujourd'hui encore il n'y a pas d'édition définitive de «La Terre vaine ». Et il n'y en aura probablement jamais. Ainsi, ce qu'Eliot entendait exactement faire dire à son grand poème doit rester ambigu dans certains de ses détails ${ }^{20}$.

Il nous faut toutefois noter que l'étude des versions publiées de cette fiction poétique extraordinaire et en partie encore philosophique a fait que certains lecteurs critiques se sont demandés de façon répétée si Pound n'aurait pas pu faire un peu mieux. En effet, comprendre suffisamment certaines des énonciations poétiques essentielles d'Eliot, déterminer leurs significations littérales, voilà qui demeure toujours problématique pour des interprètes réfléchis quelque soixante-dix ans après la première publication du poème et sa reconnaissance en tant que chef-d'œuvre du modernisme littéraire ${ }^{21}$. Et certaines de ces énonciations cruciales dépendent des usages variés du mot "rien ", un mot important non seulement dans la poésie littéraire moderniste, mais aussi dans la vie.

Avec au moins ces éléments du contexte présents à l'esprit, il nous faut maintenant revenir au détail des constructions sommaires de Davidson quant à une explication standard de la signification littérale.

20. Collected Poems 1909-1962, London, Faber and Faber, 1963, p. 67. On ne peut connaître la manière exacte dont Eliot voulait finalement que soient espacés et ponctués certains des vers en vue d'une impression définitive correcte. Comme nous le verrons, ces questions apparemment pédantes ne sont pas en réalité sans conséquences pour saisir le sens littéral supposé ainsi que l'importance des lignes citées. Pour les problèmes compliqués liés à la publication et au texte, voir North, 2001, p. xi-xiii. North inclut aussi une chronologie, des bibliographies et des sélections des sources considérables d'Eliot, les premières réactions et recensions critiques, et de nouvelles études récentes.

21. La prétention du poème à être considéré comme un chef-d'œuvre littéraire est étroitement liée en partie à la façon extraordinaire dont il réussit à exprimer ce que l'ami d'Eliot, J. M. Keynes, a appelé la «torpeur atroce ", « le terrible épuisement ", "la convulsion désespérante" de cette époque dans sa critique prophétique du traité de Versailles. Eliot avait attentivement étudié Les Conséquences économiques de la paix (1919) de Keynes. Ainsi, "La Terre vaine" peut être comprise, a écrit très récemment le poète Tom Paulin, comme un poème keynésien qui résulte de la désintégration aussi bien économique que culturelle de l'Europe dans la période de l'immédiate après-guerre " «Many Cunning Passages : How Maynard Keines Made His Mark on the "Waste Land” ", TLS, 29 novembre 2002, p. 14). 


\section{Significations littérales}

Davidson n'a pas prêté aux œuvres de l'art verbal l'attention soutenue et détaillée que leur ont consacrée d'autres philosophes contemporains. Mais il s'est largement occupé des questions de sens et de signification. De manière générale, ses intérêts ont davantage porté sur la linguistique et la logique que sur l'esthétique et la poésie ${ }^{22}$.

Toutefois, dans plusieurs essais sur la métaphore, la communication et « les limites du littéral », Davidson a considéré attentivement certaines particularités métaphoriques et stylistiques qui surviennent largement dans différents usages de la langue, spécialement dans les œuvres littéraires ${ }^{23}$. On doit trouver derrière ces essais les questions plus générales qui ont engagé Davidson pour de nombreuses années dans ses tentatives de consolidation et finalement de dépassement de l'œuvre de son maître, W. V. Quine ${ }^{24}$, tout en s'opposant notablement au travail très différent de son distingué contemporain, Michael Dummett.

On peut toujours considérer que les intérêts centraux de Davidson comprennent sa double réponse à cette question : "Qu'est-ce que cela veut dire pour les mots que de signifier ce qu'ils signifient? " Pour Davidson, on peut l'exprimer en décrivant de façon informelle ${ }^{25}$ ce que sait implicitement quelqu'un qui comprend ces mots - ce qu'un interprète raisonnable de la séquence poétique d'Eliot "La Terre vaine" sait implicitement par exemple des usages d'expressions telles que "rien », ce qu'il sait, pour ainsi dire, à partir de son expérience personnelle et de sa consultation, mettons, du Oxford English Dictionary intégral.

Davidson pense qu'il faut une théorie pour fournir cette description de ce que cela veut dire pour les mots de signifier ce qu'ils expriment. Cette théorie reconnaîtrait « la nature holiste de la compréhension linguistique »

22. Pour une présentation approfondie des principales perspectives d'une bonne partie de l'œuvre plus tardive de Davidson, voir Max Koelbel, «Two Dogmas of Davidsonian Semantics », Journal of Philosophy, 98, 2001, p. 613-635. Entre autres personnes, Koelbel remercie Davidson lui-même pour d'"utiles commentaires ".

23. Voir, par exemple, les essais dans R. W. Dasenbrock, dir., Literary Theory After Davidson, University Park (PA), Penn State University Press, 1993, spécialement l'introduction, p. 1-17. Pour une excellente discussion de l'œuvre de Davidson en ce qui concerne à la fois l'interprétation et la traduction, voir M. Montminy, Les fondements empiriques de la signification, Montréal, Bellarmin, 1998, p. 97-152. Le travail de Martin Montminy m'a été très utile, et je le remercie tout spécialement de sa générosité pour avoir examiné aussi attentivement que patiemment des ébauches antérieures de cet essai.

24. Pour des réflexions récentes sur Davidson et Quine voir L Bergstrom, «Davidson's Objections to Quine's Empiricism », et F. Tersman "Davidson and Quine's Empiricism », dans P. Kotatko, P. Pagin et G. Segal, dir., Interpreting Davidson, Stanford, CSLI, 2001, respectivement p. 17-36 et p. 269-284. Davidson répond à chacun d'eux dans le même volume, p. 290-292.

25. Pour un traitement plus formel de la "théorie compositionnelle de la signification » de Davidson, voir E. Lepore, et K. Ludwig, «What is Logical Form ? » dans Kotatko et al., dir., Interpreting Davidson, surtout p. 117-122. 
en fournissant une "interprétation de toutes les affirmations réelles ou possibles d'un locuteur ou d'un groupe de locuteurs ». Il faudrait aussi une théorie qui «serait vérifiable sans connaissance des attitudes propositionnelles détaillées du locuteur », c'est-à-dire une théorie du dire, du croire, du savoir, du désirer et ainsi de suite, qui ne dépendrait pas des concepts mêmes qu'elle a pour dessein d'expliquer ${ }^{26}$.

Dans cette théorie, toutefois, c'est la vérité plutôt que la signification qui doit être considérée comme la notion primitive. À partir d'une explication de la vérité fondée sur l'idée de conditions de vérité, il faut s'efforcer de parvenir à une compréhension de la signification. De plus, la vérité étant prise comme primitive, le modèle pour la structure de la vérité inclurait, parmi d'autres éléments, une appropriation critique de traits centraux de la sémantique formelle de Tarski, la relation de satisfaction entre entités et expressions, les caractéristiques "décitationnelles» des prédicats de la vérité, «le schéma des assentiments aux phrases ", une maximisation "juste» d'un accord entre théories concurrentes de l'interprétation (principe de charité) et une réfutation anti-empiriste des dualismes entre schéma et contenu.

Il semblerait donc que l'expression de ce qu'un interprète raisonnable sait implicitement des emplois conversationnels de certaines expressions cruciales comme "rien » ne requiert pas simplement une réflexion sur ses propres pratiques linguistiques et un bon dictionnaire. Elle paraîtrait aussi exiger l'édification d'une théorie descriptive holiste de la compréhension linguistique fondée sur la vérité — rien de moins.

Bien qu'une large part de ce travail continuel demeure plutôt technique, des essais plus tardifs de Davidson examinent en des termes plutôt directs certaines particularités du langage, y compris un petit nombre de ses usages poétiques. Un exemple important en est l'essai de 1986, A Nice Derangement of Epitaphs, avec lequel nous avons commencé.

\section{Le sens littéral comme «sens premier»?}

Davidson y insiste au début sur la distinction entre le «sens premier» ou littéral des expressions et tout «sens second» ou non littéral (que nous interprétions ce sens second comme «sens du locuteur» ou autrement). Il faut toutefois noter que littéral et non littéral ne doivent pas être compris strictement comme deux espèces de la signification, puisque la signification non littérale est pour Davidson un aspect de l'usage.

Davidson pense que nous pouvons voir le bien-fondé qu'il y a à appeler la signification littérale "sens premier" si nous réfléchissons à ce qu'il nous faut savoir afin d'expliquer certaines images en poésie. Comme exemple de cette réflexion, il nous demande de considérer ce dont nous avons besoin 
pour expliquer l'image déterminante de ces vers magnifiquement significatifs mais toujours mystérieux du sonnet 53 de Shakespeare :

Speak of the spring and foison of the year,

The one doth shadow of your beauty show,

The other as your bounty doth appear ${ }^{27}$...

Parle-t-on du printemps, de l'abondance de l'année?

L'un, de votre beauté ne nous montre que l'ombre,

L'autre veut refléter votre riche largesse...

Quelque non littérale que puisse être la signification de ces lignes, Davidson prétend que le lecteur ne peut pas saisir cette signification seconde non littérale sans "d'abord» comprendre la signification littérale du dictionnaire, "basée sur l'usage réel » à l'époque de Shakespeare, du mot foison (maintenant glosé, nous l'avons vu plus haut dans ma note précédente, comme "récolte ou moisson abondante»).

Cet exemple ne va pourtant pas sans difficultés.

Je crois que Davidson sous-estime précisément ici la difficulté qu'il y a à comprendre la signification de l'usage courant au temps de Shakespeare donnée par le dictionnaire ${ }^{28}$.

27. Pour une collation des manuscrits, une édition et un commentaire du sonnet complet dans un texte à l'orthographe modernisée, voir The Complete Sonnets and Poems, C. Burrow, dir., Oxford, Oxford University Press, 2002, p. 486-487.

Davidson cite les vers 9 à 11, qui composent grammaticalement dans le texte, quoique de façon indépendante, une phrase incomplète. Le vers 12, qui n'est pas cité : And you in every blessed shape we know ( "Nous ne voyons que vous en chaque forme exquise ») complète la phrase dans le texte.

Burrow glose l'expression foison au vers 9 , par « récolte ou moisson abondante » à partir de l'OED. Il transpose le vers 10 : The one doth shadow of your beauty show ( «L'un, de votre beauté ne nous montre que l'ombre») en The spring is merely an image of your beauty («Le printemps n'est qu'une simple image de votre beauté»). Il reprend le vers 11 : The other as your bounty doth appear ("L'autre veut refléter votre riche largesse») par The bountiful harvest seems like your generosity ( "La moisson généreuse s'apparente à votre générosité »). Et il glose le know au vers que Davidson ne cite pas par recognize ( «reconnaître»).

Notons que, après ces substitutions, nous nous retrouvons avec une phrase complète dans le texte qui n'est bien sûr rien de plus que la médiocre poésie de Burrow :

Speak of the spring and [plentiful crop] of the year,

[the spring is merely an image of your beauty,]

[the bountiful harvest seems like your generosity,]

And you in very blessed shape we [recognize].

«Parle-t-on du printemps et de [la récolte abondante] de l'année,

[le printemps n'est qu'une simple image de votre beauté,]

[la moisson généreuse s'apparente à votre générosité,]

Et nous vous reconnaissons en toute forme bénie. »

28. La tâche de Davidson est bien difficile. Voir A. Schmidt, Shakespeare Lexicon and Quotation Dictionary, $3^{\mathrm{e}}$ éd., 2 vol., Berlin, 1902; et A. C. Partridge, A Substantive Grammar of Shakespeare's Nondramatic Texts, Charlottesville, University of Virginia Press, 1976. 
La détermination, par exemple, des "significations du dictionnaire » relatives aux usages de certaines expressions-clés dans les sonnets de Shakespeare suppose davantage qu'une simple consultation des citations dans l'actuelle seconde édition de l'Oxford English Dictionary) en 20 volumes. En effet, cet ouvrage de référence, quoique standard, date maintenant nettement, et il est prévu de le remplacer dans les dix prochaines années par une édition totalement nouvelle qui ne comprendra pas moins de 40 volumes. De plus, saisir ne serait-ce que les aspects lexicaux de l'usage shakespearien dans les sonnets de 1609 suppose aussi de comprendre les usages tout à fait connexes d'expressions conventionnelles essentielles dans plus de vingt séries de sonnets publiées entre 1592 et 1606 environ $^{29}$.

La compréhension de la signification donnée par le dictionnaire aux nombreuses unités lexicales shakespeariennes requiert de surcroît l'intelligence des particularités de la syntaxe shakespearienne. Ce n'est pas chose facile malgré la disponibilité des grammaires shakespeariennes déjà réalisées.

Que dire aussi de la pragmatique de l'usage shakespearien, des jeux extraordinaires de ses expressions dans les attitudes langagières propres aux conventions du théâtre élisabéthain? Pouvons-nous être sûrs d'avoir compris les «significations du dictionnaire » d'expressions shakespeariennes difficiles à éclaircir, même après avoir mené des recherches approfondies sur les items lexicaux et appris la syntaxe?

Ce qui est plus important que la sous-estimation de la difficulté qu'il y a à comprendre certaines significations littérales comme "significations premières, significations du dictionnaire », je crois que Davidson ne prend pas ici assez au sérieux les types très différents d'images que l'on rencontre dans beaucoup d'énoncés.

Que ce soit chez Shakespeare, Sheridan ou Eliot, saisir par exemple les significations non littérales des échanges verbaux dans certaines représentations poétiques semble ne pas seulement requérir quelque chose de plus que la compréhension première de la signification appropriée du dictionnaire correspondant au mot qui exprime l'image. Il peut se faire au contraire que cette saisie requière d'abord quelque chose de radicalement différent de la compréhension des significations du dictionnaire.

Nous pouvons peut-être mieux nous rendre compte de ce point littéraire critique en prenant certains éléments d'un commentaire de la fameuse image d'Eliot dans un de ses premiers poèmes "La Chanson d'amour de J. Alfred Prufrock ». L'image en question est celle utilisée par Eliot pour rendre un soir particulier que le poète décrit comme "... étendu contre le ciel / Comme un patient anesthésié sur une table» (...spread out against the sky / Like a patient etherised upon a table).

Saisir ici la signification littérale du mot etherised - le liquide volatile incolore $\left(\mathrm{C}_{2} \mathrm{H}_{5}\right)_{2} \mathrm{O}$ - suppose en réalité autre chose que de saisir "d'abord » la signification du dictionnaire fondée sur l'usage réel au temps d'Eliot.

29. Voir la liste dans Burrow, 2002, p. 168-169. 
Il faut plutôt reconnaître "d'abord » que l'usage de l'expression etherised n'est pas «observationnel» au sens littéraire des critiques - nous ne pouvons pas à proprement parler visualiser cette comparaison : l'usage de l'expression est "symptomatique ». Comme le poète et critique Stephen Spender l'a expliqué il y a de nombreuses années en commentant cette image, justement, l'usage symptomatique du mot etherised évoque "la combinaison des connotations cliniques et romantiques [que suggère] l'état de conscience suspendu du "patient" et la tête du rêveur pleine du ciel nocturne et des étoiles ${ }^{30}$ ».

Ainsi, bien que l'exemple qu'il emprunte à Shakespeare puisse en partie clarifier ici le discours que tient Davidson sur le littéral en tant que signification première, sa discussion de l'exemple shakespearien ne suffit pas pour caractériser la signification première de façon pleine et entière. En effet, comme Davidson lui-même le fait ressortir par la suite en minant partiellement par là sa propre position, certains lecteurs déterminent souvent la première signification sans du tout commencer par prendre un dictionnaire.

Cela signifie que certains lecteurs se contentent parfois de repérer «ce à quoi le locuteur voulait en venir » et déterminent alors le sens littéral; d'autres fois, ils «trouvent l'image et découvrent ainsi la signification première» (p. 435). $\mathrm{Ou}$, comme nous venons de le voir dans le cas de "Prufrock », ils savent comment les images poétiques peuvent provoquer différents effets et ils continuent simplement à inférer le sens premier.

Ainsi, si j'ai bien compris Davidson, l'esquisse initiale de la distinction qu'il propose entre sens premier ou littéral et sens second ou sens du locuteur a besoin d'une élaboration ultérieure. Et Davidson lui-même entreprend immédiatement ce nouveau travail.

\section{Le sens littéral comme «signification linguistique de principe»?}

Après avoir exploré d'autres approches pour distinguer le sens littéral comme sens premier du sens dérivé, en faisant appel par exemple aux intentions du locuteur et à la capacité qu'a l'auditeur de comprendre ces intentions, Davidson en vient à une distinction connexe qui ne se situe plus maintenant entre le sens littéral ou premier, et le sens non littéral ou sens second, mais entre la signification linguistique et la signification non linguistique.

Il propose trois principes qui semblent caractériser la signification spécifiquement linguistique par opposition à la signification non linguistique de signes ou de signaux (p. 436). (Ces trois principes correspondent aux points trois, quatre et cinq de la caractérisation initiale de l'explication standard de la signification littérale vue plus haut.) Et il semble alors identifier cette signification linguistique de principe avec ce qu'il avait antérieurement appelé "signification première ".

30. S. Spender, p. 42. 
Ainsi, la signification littérale comme signification linguistique de principe est maintenant considérée comme : 1) systématique ( «il doit y avoir des relations systématiques entre les significations des énonciations »);2) partagée (il faut qu'une méthode d'interprétation de ces relations soit partagée entre un locuteur et un auditeur); 3) préparée (c'est-à-dire que la connaissance que le locuteur et l'auditeur ont de ces relations « est acquise avant les occasions d'interprétation et possède un caractère conventionnel»).

Tout cela est utile tant que ça fonctionne. Mais les principes de la signification linguistique qui sembleraient permettre à Davidson de caractériser la signification première comme signification linguistique de principe s'avèrent receler leurs propres difficultés. Nous pouvons être ici très bref dans la mesure où il se révèlera plus utile de détailler certaines de ces difficultés plus bas plutôt qu'ici.

Si nous reconnaissons qu'il y a des difficultés liées à ces principes, cette distinction supplémentaire entre la signification littérale comme signification linguistique de principe et la signification non littérale comme signification non linguistique est alors problématique, et ce, quelles que soient ses relations avec la première distinction entre la signification littérale comme signification première et la signification non littérale comme signification seconde.

\section{La signification littérale comme «significations coïncidentes »?}

Comme s'il reconnaissait la nécessité d'un travail toujours plus important quant à la nature exacte de la signification littérale, Davidson continue ses commentaires sur le sujet en faisant appel à une troisième distinction. Celleci toutefois n'est pas à établir entre deux sortes de significations, première et seconde, ou linguistique et non linguistique. Elle se situe plutôt entre deux types de théories dont Davidson suppose qu'elles sont requises par les « interprètes» (comme il continue à appeler tant les locuteurs que les auditeurs) pour une communication effective.

Cette distinction supplémentaire que nous avons déjà vue différencie les théories préalable et passagère. Mais rendre explicite cette distinction entre théorie préalable et théorie passagère suppose maintenant d'adopter ce que Davidson appelle alors sa "proposition simplifiée et idéalisée » quant à ce qui se passe dans la communication linguistique.

Dans la communication linguistique prise de façon générale, Davidson croit qu'il y a d'un côté "un interprète [auditeur, lecteur] qui possède à tout moment d'une transaction verbale » une théorie antécédente ou "préalable». L'interprète modifie cette théorie antécédente lorsque son interlocuteur parle dans le cadre d'une théorie ultérieure ou " passagère ». C'est-à-dire que pendant que l'interlocuteur parle, l'interprète "formule des hypothèses à propos de nouveaux noms, en modifiant l'interprétation de prédicats familiers et en revoyant les interprétations passées d'énonciations particulières à la lumière de nouvelles données» (p. 441). 
De l'autre côté, «la vision par le locuteur [ou celui qui écrit] de la théorie préalable de l'interprète $[. .$.$] est une part importante de ce sur quoi il doit$ se fonder s'il veut être compris. » Ainsi :

Pour l'auditeur la théorie préalable exprime la façon dont il est préparé à l'avance pour interpréter une énonciation de celui qui parle, tandis que la théorie passagère est la façon dont il interprète vraiment l'énonciation. Pour celui qui parle, la théorie préalable est ce qu'il croit être la théorie préalable de l'interprète, tandis que sa théorie passagère est la théorie qu'il soubaite que l'interprète utilise (p. 442 ; c'est Davidson qui souligne).

Le point essentiel ici, c'est que la théorie passagère a besoin d'être partagée parce que sans ce partage la compréhension ne peut être complète. Toujours est-il que la théorie passagère partagée ne doit pas être identifiée de quelque façon générale que ce soit avec la compétence linguistique, par exemple. Pourtant, la théorie passagère partagée décrit vraiment la compréhension des mots de celui qui parle (en cette occasion) parce qu'elle inclut une variété d'items particuliers qui sont le résultat d'une occasion particulière seulement.

La théorie passagère partagée ne doit pas en outre être identifiée avec une théorie d'une «langue naturelle réelle » à l'instar du français ou de l'anglais. Savoir en effet comment interpréter dans une occasion particulière ne comporte pas la garantie que l'on saurait comment le faire dans une autre circonstance. La théorie passagère est plutôt le point où la compréhension et l'accord s'efforcent de coïncider dans une saisie des significations.

C'est justement ici, avec la compréhension de ces significations coïncidentes comme significations littérales ou premières, que Davidson fait converger ses distinctions. "Toute déviation de l'usage ordinaire, écrit-il, pour autant qu'on en convienne pour le moment (que l'usage soit déviant ou non en connaissance de cause, d'un ou des deux côtés), se trouve dans la théorie passagère comme une caractéristique de ce que les mots signifient à cette occasion. De telles significations, pour passagères qu'elle puissent être, sont littérales, elles sont ce que j'ai appelé les significations premières » (p. 442-443).

Cet examen détaillé est certes utile. Davidson prend en outre la peine de le nuancer attentivement et affirme que ses propositions sont simplifiées et idéalisées. Toujours est-il qu'il nous faut noter une fois encore, comme nous l'avons fait avec les commentaires précédents sur les images, que l'utilisation de cette discussion fait ressortir plusieurs questions intéressantes pour l'élucidation du cas dont nous traitons - parvenir à comprendre correctement les usages littéraux de l'importante expression centrale "rien » dans la représentation poétique et dramatique offerte par Eliot d'un dialogue conversationnel incomplet.

Le discours où l'auditeur est vu comme «interprète » doit être distingué du discours où c'est le lecteur critique qui est vu comme tel. Ici, de plus, la description de ce que fait normalement l'auditeur dans une conversation ordinaire n'a pas pour but de rendre la situation complexe du dialogue poétique 
fictif où l'auditeur représenté n'est pas tant en train de décoder ce que la femme est représentée comme étant en train de dire- en changeant ses théories antérieures et passagères tandis que continue la «transaction spéciale »que de répondre silencieusement (dans sa tête) à une situation par des phrases inexprimées et des citations tirées d'une situation complètement différente. Finalement, toute discussion relative au partage d'une théorie passagère sert ici à souligner le caractère radicalement fragmenté, brisé et incomplet de certains échanges linguistiques réels et pas seulement imaginés, comme ceux représentés dans notre extrait de «La Terre vaine».

\section{Significations littérales et expressions déviantes}

Quand nous centrons notre attention sur les usages déviants de la langue tels que les impropriétés de langage, de nouveaux problèmes deviennent immédiatement évidents quant à cette caractérisation de la signification littérale comme signification première. En effet, les énonciations déviantes en général et les impropriétés de langage en particulier (pouvons-nous ajouter certains usages non standard des négations?) "introduisent des expressions qui ne sont pas couvertes par l'apprentissage antérieur, comme les expressions familières qui ne peuvent être interprétées par aucune des compétences discutées jusqu'ici» (p. 457). Le problème ne porte donc pas maintenant sur les aspects systématiques et partagés de la signification littérale, mais sur la conjonction de ces aspects avec la nature nouvelle de la signification de ces expressions, c'est-à-dire le fait que ces significations ne soient pas "préparées ", apprises à l'avance.

Pour résoudre ce problème, Davidson modifie les trois principes de la signification linguistique littérale que nous avons notés brièvement plus haut de sorte qu'ils puissent, en étant modifiés, s'adapter aux impropriétés de langage et autres expressions déviantes. (Notons toutefois que Davidson ne modifie pas ici sa distinction ambiguë entre théories préalable et passagère.) Le changement-clé, pense-t-il, consiste à invoquer un élément technique, "selon les orientations données par Tarski d'une définition de la vérité » comme moyen d'expliquer précisément comment l'on pourra dire qu'un " interprète " possède un système pour interpréter les énonciations potentiellement illimitées de phrases nouvelles.

Qu'est donc censée permettre une telle définition? La définition de la vérité de Tarski «fournit une caractérisation récursive des conditions de vérité de toutes les énonciations possibles du locuteur, et elle le fait à travers une analyse des énonciations en termes de phrases constituées à partir du vocabulaire fini et du stock fini des modes de compositions» (p. 437-8).

Mais, comme je l'ai noté plus haut de façon anticipée, une fois que les difficultés variées relatives au traitement des impropriétés de langage ont été explorées, la conclusion de Davidson s'avère très surprenante. Il conclut que les deux premiers principes de compréhension de la signification linguistique littérale — le fait que le sens premier doit être systématique et partagé — 
peuvent être sauvés, de "manière plutôt inhabituelle" seulement (p. 446). Quant au troisième principe - le fait que le sens premier doit être préparé on ne peut pas le sauver du tout.

Le troisième principe doit donc être abandonné. Mais l'abandon de ce principe semble impliquer aussi celui de l'idée antérieure de la signification littérale comme signification linguistique de principe. Et, puisque cette idée dépend étroitement de la notion-clé de signification littérale comme signification première, l'abandon de l'idée de signification littérale comme signification linguistique de principe semble impliquer aussi celui de l'idée de signification littérale comme signification première ${ }^{31}$.

La conclusion particulière semble donc que nous ne disposions toujours pas d'une explication satisfaisante de la signification littérale. Et la conclusion plus générale semble être que «dans la communication linguistique rien ne corresponde à une compétence linguistique » comme Davidson et tant d'autres philosophes et linguistes l'ont décrite.

Ces conclusions, pour provisoires qu'elles soient, constituent un contretemps majeur pour résoudre ou faire en sorte que s'évanouisse le problème le plus général de Davidson — « je veux savoir, écrit-il, comment les gens qui ont déjà un langage [...] réussissent à appliquer leur compétence ou leur connaissance à des cas réels d'interprétation [...] Mon problème est de décrire ce qui est impliqué dans l'idée "de posséder un langage" [...]” (p. 441).

\section{Signification littérale et compréhension linguistique holistique}

Face à ces conclusions inattendues et décevantes, je pense qu'il faut revenir sur plusieurs points. Cette explication, nous nous en souvenons, provient d'un intérêt très général visant à exprimer la manière dont les locuteurs compétents d'une langue réussissent à s'interpréter mutuellement. La question particulière est ici de savoir quelle serait une explication satisfaisante de ce que cela veut dire «pour les mots de signifier ce qu'ils signifient ». Une telle explication serait suffisamment complète pour inclure la compréhension d'expressions déviantes n'incluant pas que des impropriétés de langage. Ces expressions déviantes, je suppose, comprendraient aussi des usages non standard d'expressions négatives polyvalentes problématiquement référentielles, à l'instar des emplois variés de l'expression « rien» dans un poème comme «La Terre vaine» d'Eliot.

31. Peut-être cet argument sommaire a-t-il besoin d'être développé. Que l'on considère dans cette perspective l'objection de M. Montminy : "Je ne vois pas pourquoi le fait de dire que l'interprétation n'est pas toujours fondée sur la signification linguistique "préparée” implique que nous devions abandonner l'idée de la signification littérale comme signification première. Les impropriétés de langage semblent être des cas où il y a une signification littérale et où la compréhension de cette signification littérale n'est pas fondée sur la compréhension d'une signification linguistique préparée. Nous pouvons ainsi rejeter la signification préparée sans abandonner la signification littérale» (communication personnelle). En réponse, je serais enclin à ce que nous explorions ensemble de manière un peu plus approfondie la question de savoir si nous sommes d'accord sur ce que sont les impropriétés de langage. 
La réponse à cette question particulière est supposée prendre la forme d'une théorie. Et cette théorie doit, entre autres tâches, être capable d'expliquer comment "des interprètes» comprennent des énonciations antérieurement inconnues du "même langage ». (La raison exacte de la nécessité de cette théorie ainsi que son sens précis ne sont pas expliqués bien clairement, malgré l'examen des caractéristiques de la définition de la vérité de Tarski.) La théorie doit en outre éviter de laisser de côté les questions de fond et reconnaître, comme nous l'avons noté auparavant, «la nature holistique de la compréhension linguistique ${ }^{32}$ ».

L'élément essentiel dans cette théorie, c'est la double primauté de la vérité par rapport à la signification et de la signification littérale par rapport à la signification non littérale. Davidson dit ici peu de choses sur la vérité, un sujet qu'il traite par ailleurs en profondeur. Il cherche plutôt à caractériser la signification littérale de manière toujours plus détaillée.

La signification littérale, nous nous en souvenons, doit être comprise plus justement comme "signification première " au sens où la compréhension de la signification littérale est un préalable à celle de la signification non littérale. Grâce à la distinction supplémentaire entre théories préalable et passagère, les déviations des cas ordinaires de la signification littérale sont subsumées par les significations littérales au sein de la théorie passagère. Mais la tentative d'explicitation du caractère systématique et partagé des significations premières par le recours à la description récursive de la vérité de Tarski est finalement vaine. Le résultat en est une curieuse notion de signification littérale comme "première » en un sens qui attend toujours une description satisfaisante.

Nous pourrions donc à peu près formuler sans injustice ces réflexions relatives à l'interprétation de la signification littérale en pensant aux expressions d'œuvres poétiques en fonction de perspectives comme celles-ci :

1. L'interprétation de ce que cela veut dire pour certains mots (et peutêtre certaines phrases) de signifier ce qu'ils signifient dans des œuvres littéraires de fiction suppose la construction d'une théorie non circulaire de la nature holistique de la compréhension linguistique qui soit suffisamment détaillée pour expliquer les expressions déviantes comme les expressions normales.

2. Dans cette théorie de la nature holistique de la compréhension linguistique, on doit considérer «la vérité " comme un terme primitif à la lumière duquel il faut expliquer la signification. Il faut en outre expliquer l'antériorité de la signification littérale au plan épistémique par rapport à la signification non littérale.

3. On doit aussi comprendre les significations littérales normales des mots (et peut-être des phrases) dans certaines expressions d'œuvres littéraires

32. On pourra trouver les discussions pertinentes à ce sujet pour l'exposé de Davidson sur le «holisme» dans J. Fodor, et E. Lepore, Holism : A Shopper's Guide, Oxford, Blackwell, 1992. 
de fiction en fonction des «théories " préalable et passagère tant de celui qui écrit (parle) que du lecteur (interprète). Les significations déviantes doivent être par contre interprétées du point de vue des significations littérales dans le cadre de la théorie passagère du lecteur (de l'interprète).

En bref, ce que cela pourrait vouloir dire pour les paroles de la femme par exemple, de signifier ce qu'elles signifient littéralement dans le dialogue représenté poétiquement par Eliot «Tu ne sais rien ?... N'as-tu donc rien dans la tête ?", voilà qui n'est pas évident. Même constat pour des lecteurs compétents qui chercheraient à comprendre ici les relations difficiles entre les significations littérale et non littérale d'une manière holistique et non circulaire, qui accorde à la notion de vérité une primauté par rapport à celle de la signification. En effet, selon Davidson lui-même demeurent au moins problématiques tant la distinction essentielle entre significations littérale et non littérale que la nécessaire théorie holistique de la compréhension linguistique.

Les présentes analyses de Davidson fournissent néanmoins, me semble-t-il, d'utiles clarifications quant à la nature de la signification littérale dans certaines représentations littéraires telles que la poésie moderniste de la souffrance d'Eliot. L'insistance sur la nature holistique de la compréhension linguistique, bien qu'elle ne soit pas expliquée ici d'une façon suffisamment claire, fait vraiment bien ressortir certaines des voies générales d'où résultent de fructueuses interprétations de séquences poétiques comme «La Terre vaine » d'Eliot, à l'instar de la saisie exemplaire des significations des images centrales par Stephen Spender.

En outre, l'attention aux «expressions déviantes» est utile elle aussi. Le fait de souligner la compréhension mutuelle réussie d' 'expressions déviantes » dans de nombreux échanges conversationnels met en relief l'un des éléments distinctifs quoique toujours problématiques des multiples emplois poétiques de la langue que des interprètes rationnels ont à exprimer. Et cela demeure le cas même si les façons précises dont on doit interpréter les supposées significations littérales des expressions déviantes nécessitent toujours un examen plus approfondi.

Enfin, la distinction initiale entre théorie préalable et théorie passagère est suggestive en ce qu'elle souligne le besoin qu'ont des interprètes rationnels de construire des descriptions d'ensemble des idiolectes de chaque poète. Ces idiolectes peuvent alors servir à faire ressortir certains usages particuliers de la langue au sein d'un poème. Il en va de même pour les usages déviants eu égard aux usages normaux au sein de l'idiolecte. Nous avons toutefois besoin ici aussi d'une analyse supplémentaire.

\section{Interprétation, significations littérales et philosophie du langage}

Pris conjointement, certains éléments de ces explications philosophiques nous fournissent vraiment d'utiles considérations pour une tentative d'interprétation de la signification d'expressions difficiles dans la poésie moderniste de la souffrance, comme pour les significations littérales de différents emplois de «rien» dans «La Terre vaine». En guise de conclusion, j'aimerais souligner plusieurs points positifs et négatifs particuliers. 
La notion d' "interprétation " d'emplois variés d'expressions dans une œuvre littéraire, comme processus centré sur certains modèles spécifiques et qui s'efforce de s'accommoder aussi bien aux intentions de l'écrivain que du lecteur, trouve un équilibre entre considérations formalistes et pragmatiques. Qu'une interprétation rationnelle doive être articulée de sorte qu'on puisse la vérifier inductivement, voilà qui est aussi une protection nécessaire contre de nombreuses lectures impressionnistes ou intuitives des œuvres littéraires.

De plus, la distinction entre intentions et attentes à long terme et à court terme, tant du côté des écrivains que des lecteurs, souligne clairement le besoin qu'il y a de cibler une interprétation rationnelle sur des aspects très spécifiques d'un phénomène extrêmement complexe. Ce phénomène peut être vu comme celui des changements de sens de ce que l'on doit précisément comprendre comme significations littérales d'énonciations poétiques.

Ce qui constitue enfin un rappel propre à faire réfléchir, c'est l'insistance sur cette explication concurrente, attentive et sophistiquée, à propos de l'explicitation des présuppositions d'une interprétation rationnelle quant à la priorité ou bien des questions sur la signification ou bien de celles concernant la vérité. Car, pour spécifiques que soient les fins d'une interprétation rationnelle, les expressions dans lesquelles l'interprétation elle-même est formulée requièrent une attention critique propre.

Ces rappels positifs s'opposent toutefois à un arrière-fond qui nécessite toujours un examen critique approfondi. Que l'on se rappelle que la question initiale à laquelle répondent ces explications — « qu'est-ce que cela veut dire pour les mots de signifier ce qu'ils signifient? " — est très générale, tandis que les questions dont traite l'interprétation rationnelle des significations littérales supposées dans le cas d'expressions extraites d'une œuvre littéraire sont bien plus spécifiques.

Cette différence suggère que certains éléments des options philosophiques en cause ne sont pas aussi pertinents qu'on pourrait le croire de prime abord pour la compréhension d'expressions cruciales dans des œuvres comme «La Terre vaine» d'Eliot. Plusieurs points essentiels demeurent embarrassants lorsqu'on ne se contente pas de confronter cette explication à une citation unique d'un sonnet shakespearien, d'une pièce de Sheridan ou d'un poème d'Eliot, mais à de longs passages tirés d'œuvres modernistes par opposition aux arrière-plans historiques d'où elles émergent.

De plus, même lorsqu'elles sont revues avec soin à la lumière d'un examen critique approfondi, ces explications de la signification littérale semblent oublier le rôle très important des émotions et des sentiments dans la compréhension des énonciations poétiques ${ }^{33}$. Dans les extraits que nous avons

33. Les composantes affectives auxquelles je fais allusion ici impliquent au moins les «émotions » et les «sentiments » qui doivent être compris de manière tout à fait distincte dans la mesure où «ils sont influencés par des systèmes neuronaux distincts» (R. J. Dolan, "Neuroscience and Psychology : Emotion, Cognition, and Behaviour ", Science, 298, 8 novembre 2002, p. 1194). 


\section{2 - Philosophiques / Printemps 2005}

examinés avec une certaine attention, une grande partie de ce sur quoi nous nous sommes finalement fixés comme étant la signification littérale de certaines expressions cruciales mais problématiques, comme "rien ", implique leur propriété forgée à dessein de susciter chez certains lecteurs de vifs états affectifs et non simplement épistémiques. Dans ce contexte, toute explication satisfaisante de la signification littérale ne peut pas ne pas se préoccuper de ces propriétés particulières des significations littérales en question.

Pour instructifs que puissent être les débats qui se poursuivent entre les partisans des deux bords à propos de la nature du littéral, expliquer de façon moins insatisfaisante ce à quoi ressemble précisément le littéral dans certains contextes normatifs semble exiger un glissement quant aux priorités philosophiques de nos recherches continuelles. Pour le moment, nous pourrions peut-être penser le décrire dans les termes ouverts finis d'un changement qui, au lieu de souligner les usages sémantiques, se concentrerait sur les usages pragmatiques du langage normatif, un changement qui irait du propositionnel au communicatif, des assertions aux questions, du métaphorique au symbolique, peut-être même des phrases aux mots ${ }^{34}$.

Copyright (C) 2003 Peter J. McCormick. Tous droits réservés.

Traduit par Stéphane Agullo.

Copyright (C) Stéphane Agullo 2003 pour la traduction française.

34. Je remercie Martin Montminy et deux référents anonymes pour leurs suggestions très utiles ainsi que Stéphane Agullo pour sa traduction attentive. 